Research Article

\title{
Clinical Efficacy of Fuzheng Guben Anticancer Decoction Combined with Taxol in Treating Ovarian Carcinoma and Its Effect on Complication Incidence
}

\author{
Pinger Li and Yinmei Lou $(\mathbb{D}$ \\ Integrated Traditional Chinese and Western Medicine, The First People's Hospital, Fuyang, Hangzhou 311400, Zhejiang, China \\ Correspondence should be addressed to Yinmei Lou; yujinxiangl@126.com
}

Received 28 September 2021; Revised 19 November 2021; Accepted 20 November 2021; Published 17 December 2021

Academic Editor: Muhammad Wasim Khan

Copyright (C) 2021 Pinger Li and Yinmei Lou. This is an open access article distributed under the Creative Commons Attribution License, which permits unrestricted use, distribution, and reproduction in any medium, provided the original work is properly cited.

\begin{abstract}
Objective. To investigate the clinical value of Fuzheng Guben anticancer decoction combined with taxol in treating ovarian carcinoma (OC). Methods. The medical records of 80 OC patients treated in the First People's Hospital of Fuyang Hangzhou (January 2018-January 2021) were retrospectively analyzed, and the patients were split into the control group and the experimental group according to the treatment regimen, with 40 cases each. Those in the control group accepted the taxol chemotherapy, and on this basis, those in the experimental group took the Fuzheng Guben anticancer decoction, so as to compare its clinical efficacy and complication incidence. Results. No statistical between-group differences in patients' general information were observed $(P>0.05)$; compared with the control group, the disease objective remission rate of the experimental group was greatly higher $(P<0.05)$; before and after treatment, the changes in $\mathrm{CD} 8^{+}$were not significant, indicating no statistically significant between-group differences $(P>0.05)$, and after treatment, $\mathrm{CD} 3^{+}, \mathrm{CD} 4^{+}, \mathrm{and}_{\mathrm{CD}} 4^{+} /$ $\mathrm{CD}^{+}$were obviously higher than before and were obviously higher in the experimental group than in the control group $(P<0.05)$; after treatment, the CA125, CA199, and CEA levels were obviously lower than before and were significantly lower in the experimental group than in the control group $(P<0.05)$; the mean survival of the experimental group was significantly higher than that of the control group (19.80 \pm 5.84 vs. $14.075 \pm 5.12$ months, $P<0.05)$; and between the two groups, the incidence rate of adverse reactions of the experimental group was remarkably lower $(P<0.05)$. Conclusion. On the basis of taxol chemotherapy, jointly applying Fuzheng Guben anticancer decoction can significantly improve the clinical efficacy of OC, help to improve patients' immune function, lower the complication incidence rate, and prolong the mean survival.
\end{abstract}

\section{Introduction}

Ovarian carcinoma (OC) is a malignant neoplastic disease that occurs in the ovaries of women, with an incidence second only to cervical cancer and endometrial carcinoma, which seriously threatens the life, health, and safety of women [1-4]. Clinically, the methods of treating OC mainly include surgical resection and chemoradiotherapy, and with the gradual and deep research of traditional Chinese medicine (TCM) in tumor treatment at the current stage, TCM is also applied for treating OC patients. Taxol can effectively inhibit tumor growth and control the condition, but chemotherapy will reduce the immunity, which, combined with the untoward drug effects, has a large effect on patients [5-8]. With the continuous development of TCM technology, Chinese herbs have been gradually used in treating OC as an adjuvant therapy to regulate body immunity and relieve the adverse reactions from taxol chemotherapy, thus having an important role in improving the clinical efficacy of OC [9-12]. Fuzheng Guben anticancer decoction has the efficacy of regulating qi flowing to invigorate blood, resolving hard mass for detumescence, invigorating qi for strengthening vital qi, and nourishing blood for consolidating body resistance, which is a good choice for 
the treatment of OC. At present, there are few studies on combining Fuzheng Guben anticancer decoction with taxol in treating OC patients. Based on this, the clinical efficacy of Fuzheng Guben anticancer decoction combined with taxol in treating $\mathrm{OC}$ and its effect on complication incidence were deeply analyzed in this study.

\section{Study Methods}

2.1. Patient Grouping. The medical records of $80 \mathrm{OC}$ patients treated in the First People's Hospital of Fuyang Hangzhou from January 2018 to January 2021 were retrospectively analyzed, and the patients were divided into the control group and the experimental group according to whether they took the Fuzheng Guben anticancer decoction, with 40 cases each. Those only treated with taxol chemotherapy were included in the control group, and those who received the combined treatment of taxol chemotherapy and Fuzheng Guben anticancer decoction were included in the experimental group. The study was approved by the ethics committee of the First People's Hospital of Fuyang Hangzhou.

2.2. Inclusion Criteria. The inclusion criteria were as follows: (1) the patients were diagnosed with OC after laboratory index inspection, imaging data, and pathological examination; (2) the patients had complete data and high treatment compliance; (3) the patients met the indications of single taxol chemotherapy; and (4) the patients and their family members signed informed consent of the study.

2.3. Exclusion Criteria. The exclusion criteria were as follows: (1) the patients had severe organ failure; (2) the patients had cognition disorders; (3) the patients were allergic to the drugs applied herein; (4) the estimated survival of the patients was less than 6 months; and (5) the patients lost to follow-up during the study.

2.4. Methods. Single taxol chemotherapy was adopted to patients in the control group as follows. Before chemotherapy, the pretreatment with dexamethasone, diphenhydramine, and $\mathrm{H} 2$ receptor antagonist was performed; in the first 3 days of chemotherapy, $120-150 \mathrm{mg} / \mathrm{mz}$ of taxol (specification: $60 \mathrm{mg}$; manufacturing: Beijing Union Pharmaceutical Factory Co., Ltd.; NMPA approval no. $\mathrm{H} 20083786)$ was administered via an intravenous drip, and at the $4^{\text {th }}$ day, $90 \mathrm{mg} / \mathrm{mz}$ of taxol was administered via abdominal cavity perfusion for 2-3 times daily. Four weeks were regarded as one course, and the patients were treated for 3 consecutive courses totally [13]. On this basis, the patients in the experimental group took the Fuzheng Guben anticancer decoction. The formula was $20 \mathrm{~g}$ of Chinese angelica, $20 \mathrm{~g}$ of debark peony root, $30 \mathrm{~g}$ of American ginseng, $30 \mathrm{~g}$ of Mongolian milk vetch root, $15 \mathrm{~g}$ of dried tangerine peel, $15 \mathrm{~g}$ of Pinellia tuber, $15 \mathrm{~g}$ of Fritillaria, $6 \mathrm{~g}$ of liquorice root, and $6 \mathrm{~g}$ of Chinese date. The herbs were decocted according to the general decocting method, and the patients daily took one dose in two split times (in the morning and the evening) when it was still warm. The follow-up time of all patients was not less than 12 months.

2.5. Observation Indicators. The patients' general information including their age, BMI, course of the disease, TNM stage, and pathological type was counted. The clinical treatment effect of patients in both groups was evaluated referring to the Response Evaluation Criteria in Solid Tumors (RECICT) [14] as follows. It was considered as complete response (CR) if the patients' lesion disappeared completely or for 4 weeks; partial response (PR) if the lesion was shrunk to over $30 \%$ for over 4 weeks; stable disease (SD) if the lesion was shrunk to less than $30 \%$ or increased by less than $20 \%$; and progressive disease (PD) if the lesion was increased by more than $20 \%$ or there were new lesions. The objective remission rate $(\mathrm{ORR})=(\mathrm{CR}+\mathrm{PR}) /$ total number $\times 100 \%$.

The immune function indicators $\left(\mathrm{CD}^{+}, \mathrm{CD}^{+}, \mathrm{CD}^{+}\right.$, and $\mathrm{CD} 4^{+} / \mathrm{CD}^{+}$) were measured by the flow cytometry assay; and by drawing patients' upper limb venous blood, their levels of tumor markers including CA125, CA199, and CEA were measured by the enzyme-linked immunosorbent assay (ELISA), with the kits purchased from Shanghai Enzyme-linked Biotechnology Co., Ltd. Through follow-up visits, the patients' mean survival was recorded, and their adverse reactions were analyzed.

2.6. Statistical Processing. The data differences were calculated with SPSS 22.0, picture drawing software was GraphPad Prism 7 (GraphPad Software, San Diego, USA), the enumeration data and measurement data were expressed by $(n(\%))$ and $(\bar{x} \pm s)$ and examined by $X^{2}$ test and $t$-test, respectively, and $P<0.05$ indicated a statistical difference.

\section{Results}

3.1. General Information. After comparing the patients' general information including age, BMI, course of the disease, TNM stage, and pathological type, the result was $P>0.05$, which indicated no great between-group difference and met the study criteria of the controlled experiment. See Table 1.

3.2. Clinical Efficacy. Between the two groups, the ORR of the experimental group was greatly higher $(P<0.05)$. See Figure 1.

3.3. Immune Function. Before and after treatment, the variation in $\mathrm{CD}^{+}$of all patients was not significant, indicating no statistical meaning in the between-group difference $(P>0.05)$. After treatment, the patients' other immune function indicators were obviously higher than before $(P<0.05)$ and were significantly higher in the experimental group than in the control group $(P<0.05)$. See Table 2 .

3.4. Levels of Tumor Markers. After treatment, the CA125, CA199, and CEA levels were obviously lower than before $(P<0.05)$ and were significantly lower in the experimental group than in the control group $(P<0.05)$. See Table 3 . 
TABLE 1: General information $(n=40)$.

\begin{tabular}{|c|c|c|c|c|}
\hline Observation indicator & Control & Experimental & $X^{2} / t$ & $P$ \\
\hline Age (years) & $51.17 \pm 8.58$ & $51.30 \pm 8.79$ & 0.067 & 0.947 \\
\hline BMI $\left(\mathrm{kg} / \mathrm{m}^{2}\right)$ & $23.24 \pm 2.11$ & $23.15 \pm 2.08$ & 0.192 & 0.848 \\
\hline Course of the disease (years) & $2.27 \pm 0.30$ & $2.31 \pm 0.32$ & 0.577 & 0.566 \\
\hline \multicolumn{5}{|l|}{ TNM stage } \\
\hline IIIa & $18(45 \%)$ & $21(52.5 \%)$ & 0.450 & 0.502 \\
\hline IIIb & $14(35 \%)$ & $13(32.5 \%)$ & 0.056 & 0.813 \\
\hline IV & $8(20 \%)$ & $6(15 \%)$ & 0.346 & 0.556 \\
\hline \multicolumn{5}{|l|}{ Pathological type } \\
\hline Serous cystadenocarcinoma & $12(30 \%)$ & $13(32.5 \%)$ & 0.058 & 0.809 \\
\hline Mucinous cystadenocarcinoma & $10(25 \%)$ & $8(20 \%)$ & 0.287 & 0.592 \\
\hline Granular cell carcinoma & $7(17.5 \%)$ & $8(20 \%)$ & 0.082 & 0.775 \\
\hline Embryonal carcinoma & $7(17.5 \%)$ & $6(15 \%)$ & 0.092 & 0.762 \\
\hline Undifferentiated carcinoma & $4(10 \%)$ & $5(12.5 \%)$ & 0.125 & 0.723 \\
\hline
\end{tabular}

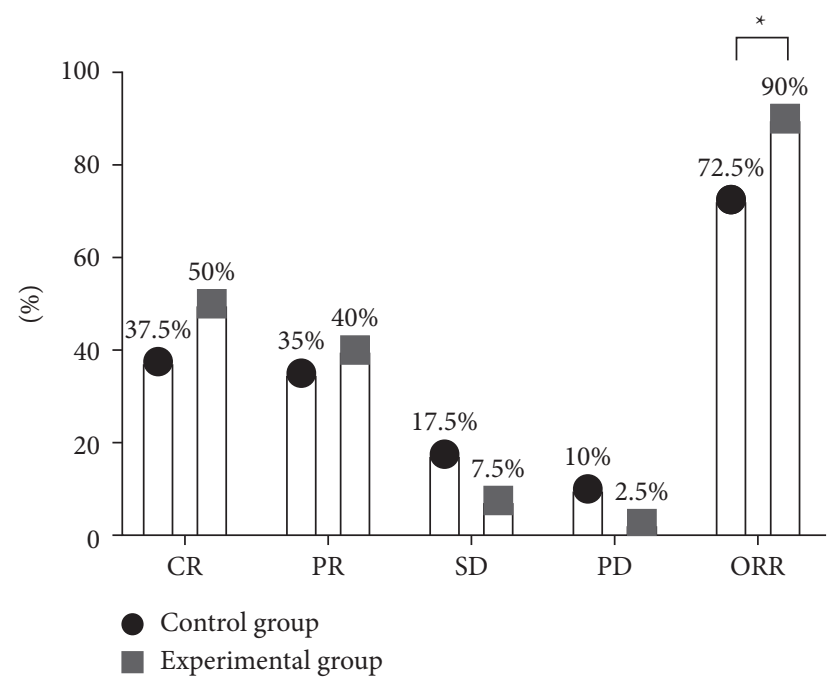

FIGURE 1: Between-group comparison of clinical efficacy. Note: the horizontal axis showed the evaluation dimensions, and the vertical axis showed the percentage. In the control group, there were $15 \mathrm{CR}$ cases, $14 \mathrm{PR}$ cases, $7 \mathrm{SD}$ cases, and $4 \mathrm{PD}$ cases, so the number of ORR cases was 29 . In the experimental group, there were $20 \mathrm{CR}$ cases, $16 \mathrm{PR}$ cases, $3 \mathrm{SD}$ cases, and $1 \mathrm{PD}$ case, so the number of ORR cases was $36 ;{ }^{*}$ indicated a significant between-group difference in ORR $\left(X^{2}=4.021, P=0.045\right)$.

TABLE 2: Between-group comparison of T-cell subset levels $(\bar{x} \pm s)$.

\begin{tabular}{|c|c|c|c|c|}
\hline T-cell subset & & Control & Experimental & $t / P$ \\
\hline $\mathrm{CD}^{+}(\%)$ & $\begin{array}{l}\text { Before treatment } \\
\text { After treatment }\end{array}$ & $\begin{array}{c}46.55 \pm 5.54 \\
52.03 \pm 5.84^{*}\end{array}$ & $\begin{array}{c}46.76 \pm 5.60 \\
57.81 \pm 6.02^{*}\end{array}$ & $4.359 /<0.001$ \\
\hline $\mathrm{CD}^{+}(\%)$ & $\begin{array}{c}\text { Before treatment } \\
\text { After treatment }\end{array}$ & $\begin{array}{c}32.74 \pm 4.82 \\
37.13 \pm 4.30^{*}\end{array}$ & $\begin{array}{c}32.86 \pm 4.85 \\
41.60 \pm 4.76^{*} \\
\end{array}$ & $4.407 /<0.001$ \\
\hline $\mathrm{CD}^{+}(\%)$ & $\begin{array}{l}\text { Before treatment } \\
\text { After treatment }\end{array}$ & $\begin{array}{l}32.60 \pm 3.29 \\
33.82 \pm 3.19\end{array}$ & $\begin{array}{l}32.44 \pm 3.18 \\
33.86 \pm 3.21\end{array}$ & $0.056 / 0.956$ \\
\hline $\mathrm{CD} 4^{+} / \mathrm{CD} 8^{+}$ & $\begin{array}{l}\text { Before treatment } \\
\text { After treatment }\end{array}$ & $\begin{array}{c}1.03 \pm 0.03 \\
1.10 \pm 0.05^{*}\end{array}$ & $\begin{array}{c}1.04 \pm 0.04 \\
1.25 \pm 0.05^{*}\end{array}$ & $13.416 /<0.001$ \\
\hline
\end{tabular}

*indicated $P<0.05$ in the comparison of patients in the same group before and after treatment.

3.5. Long-Term Efficacy. The mean survival of the experimental group was significantly higher than that of the control group $(19.80 \pm 5.84$ vs. $14.075 \pm 5.12$ months, $t=4.544, P<0.001)$. See Figure 2 for the survival curves.
3.6. Adverse Reactions. Between the two groups, the incidence rate of adverse reactions in patients was significantly lower in the experimental group $(P<0.05)$. See Table 4. 
TABLE 3: Between-group comparison of levels of tumor markers $(\bar{x} \pm s)$.

\begin{tabular}{lcccc}
\hline Test indicator & & Control & Experimental & $t / P$ \\
\hline \multirow{2}{*}{ CA125 $(\mathrm{U} / \mathrm{ml})$} & Before treatment & $90.86 \pm 14.55$ & $90.57 \pm 14.33$ & $33.02 \pm 10.13^{*}$ \\
\hline \multirow{2}{*}{ CA199 (IU/L) } & After treatment & $55.83 \pm 10.62^{*}$ & $51.02 \pm 6.71$ & $9.829 /<0.001$ \\
\hline \multirow{2}{*}{ CEA $(\mathrm{ng} / \mathrm{ml})$} & Before treatment & $50.86 \pm 6.64$ & $21.75 \pm 4.39^{*}$ & $9.568 /<0.001$ \\
& After treatment & $30.91 \pm 4.17^{*}$ & $27.17 \pm 4.79$ & \\
& Before treatment & $27.25 \pm 4.88$ & $16.02 \pm 3.07^{*}$ & $6.155 /<0.001$ \\
\hline
\end{tabular}

*indicated $P<0.05$ in the comparison of patients in the same group before and after treatment.

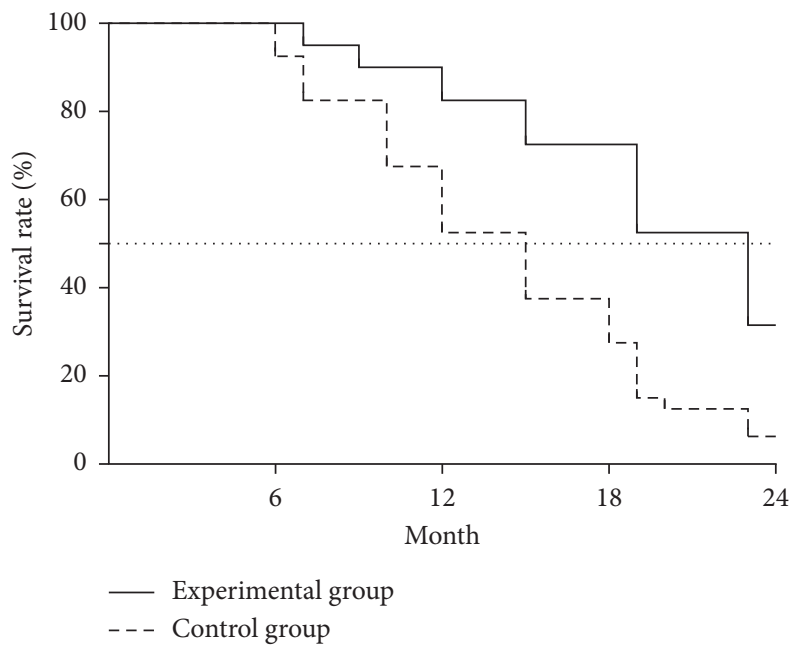

FIgURE 2: Survival curves.

TABLE 4: Adverse reaction incidence ( $n(\%))$.

\begin{tabular}{lccc}
\hline Adverse reaction & Control & Experimental & $X^{2}$ \\
\hline Neurotoxicity & $2(5)$ & $0(0)$ & \\
Reduction of the white blood cell count & $3(7.5)$ & $1(2.5)$ & \\
Nausea and vomiting & $6(15)$ & $3(7.5)$ & \\
Anemia & $4(10)$ & $0(0)$ & \\
Joint and muscle pain & $5(12.5)$ & $1(2.5)$ & \\
Thrombocytopenia & $4(10)$ & $7(5)$ & 15.221 \\
Total incidence rate & $24(60)$ & $7(17.5)$ & $<0.001$ \\
\hline
\end{tabular}

\section{Discussion}

With the development of China's society and economy, people's standard of living has been increasing and so has the life, work, and other stresses in women, and combined with objective factors such as environmental pollution, the number of new OC cases is rising year by year, and more and more young women also suffer from the disease, which has a fatality rate ranking the first among malignant tumors of the female reproductive system [15-18]. Currently, chemotherapy is the main means for the treatment of cancer, mainly improving patient symptoms and prolonging the survival through Western medicine [19-22]. Taxol is a natural anticancer drug extracted from Chinese yew. It exerts antitumor effects by interfering with the microtubule network essential for cell function in mitosis and interphase and can inhibit cell-cycle microvascular protein depolymerization, cell division, and consequently cell metabolism; in addition, it is metabolized through the liver, has little renal toxicity, and is significantly less toxic than platinum-based chemotherapy. In the study conducted by Guoqiang et al. [23] and Jiwon et al. [24], taxol has better antitumor effect in diseases such as breast cancer, lung cancer, and ovarian cancer. From the perspective of TCM, OC belongs to the category of "abdominal mass," which is mostly caused by weakness of the body, insufficient essence and blood of the liver and kidney, internal binding of phlegm turbidity and stasis toxin, etc., and chemotherapy can cause loss of yin, yang, blood, and qi, blood flow blockage, and weak organs in patients and even symptoms such as incoordination between the spleen and stomach and qi-blood loss in some cases. TCM believes that treating OC shall comply with the principle of strengthening body resistance and restoring vital energy, which shall focus on both warming the kidney and promoting blood circulation. In this study, the Fuzheng Guben anticancer decoction contained Chinese angelica 
(nourishing yin for regulating menstruation and promoting blood circulation for relieving pain), debark peony root (tonifying blood for consolidating body resistance), American ginseng and Mongolian milk vetch root (nourishing the spleen for invigorating qi and warming the kidney for reinforcing yang), dried tangerine peel (regulating qi flowing for invigorating the spleen and regulating the spleen-stomach for dispelling dampness), Pinellia tuber (relieving oppression for resolving hard mass), Fritillaria (resolving masses for detumescence), liquorice root (clearing heat and removing toxicity, invigorating the spleen-stomach, and replenishing qi), and Chinese date (regulating the spleen-stomach and nourishing qi, tonifying blood, and invigorating the spleen). Combining all the herbs could exert the efficacy of replenishing qi, tonifying blood, invigorating the spleen and stomach, and warming the kidney for reinforcing yang. This formula is quite valued in the field of TCM against cancer, and a large number of animal experimental studies have been done on its anticancer effects in the international medical community. It was reported that, in a set of induced bladder cancer experiments, rats fed with Fuzheng Guben anticancer decoction were in a better state than the others, and this formula also showed a marked effect on liver cancer metastasis in experimental mice, presenting less side effects, the function of enhancing the immune function of patients, and an anticancer role.

In this study, the ORR was greatly higher in the experimental group than in the control group $(P<0.05)$, which was consistent with the study report of Yiming and Huijun [25], implying that applying Fuzheng Guben anticancer decoction on the basis of taxol chemotherapy could effectively improve the efficacy of OC and was good for controlling the condition. Before and after treatment, the variation in $\mathrm{CD}^{+}$of all patients was not significant, with no statistically meaningful between-group difference $(P>0.05)$; after treatment, the patients' other immune function indicators were obviously higher than before and were significantly higher in the experimental group than in the control group $(P<0.05)$. OC patients are often accompanied by immune dysfunction, which in turn is one of the important factors leading to disease progression, and among the immune function indicators, $\mathrm{CD}^{+}$in $\mathrm{T}$ lymphocytes can assist other immune cells to exert antitumor effects. This result suggested that the combined regimen could greatly strengthen the immunity and promote the recovery of OC patients, and the reasons were as follows: Fuzheng Guben anticancer decoction could alleviate the damage of taxol chemotherapy on patients' immunity, reduce the body damage, and protect patients' immune function; modern pharmacology indicated that astragalin and saponin from Mongolian milk vetch root could promote the body to synthesize proteins, improve immunity, and enhance cell antitumor activity; in addition, American ginseng was rich in polysaccharide, which could induce tumor cell apoptosis. After treatment, the patients' CA125, CA199, and CEA levels were obviously lower than before and were significantly lower in the experimental group than in the control group $(P<0.05)$, demonstrating that Fuzheng Guben anticancer decoction could effectively reduce the high expression of tumor markers in pathological tissue and serum of OC patients. According to the analysis on longterm efficacy, compared with the control group, the experimental group achieved significantly higher mean survival $(19.80 \pm 5.84$ vs. $14.075 \pm 5.12$ months, $P<0.05)$ and lower incidence rate of adverse reactions $(P<0.05)$, implying that Fuzheng Guben anticancer decoction could consolidate body resistance, cultivate the primordial spirit, and effectively reduce the adverse reactions caused by taxol.

In conclusion, jointly applying Fuzheng Guben anticancer decoction on the basis of taxol chemotherapy significantly improves the clinical efficacy, promotes immune function, reduces the incidence of complications, and prolongs the mean survival. However, because the sample size of this study was small, the conclusions obtained herein still need to be confirmed by a large-sample randomized double-blind trial; the study only intervened some patients who met the indication of single taxol chemotherapy, so it has certain limitations, and the effect of combining the TCM formula with other chemotherapeutic agents still needs to be explored.

\section{Data Availability}

The data used to support the findings of this study are available from the corresponding author upon reasonable request.

\section{Conflicts of Interest}

The authors declare no conflicts of interest.

\section{References}

[1] L. Cai, G. Xu, C. Shi, D. Guo, X. Wang, and J. Luo, "Telodendrimer nanocarrier for co-delivery of paclitaxel and cisplatin: a synergistic combination nanotherapy for ovarian cancer treatment," Biomaterials, vol. 37, pp. 456-468, 2015.

[2] U. A. Matulonis, M. W. Sill, V. Makker et al., "A randomized phase II study of cabozantinib versus weekly paclitaxel in the treatment of persistent or recurrent epithelial ovarian, fallopian tube or primary peritoneal cancer: an NRG Oncology/ Gynecologic Oncology Group study," Gynecologic Oncology, vol. 152, no. 3, pp. 548-553, 2019.

[3] H. Yao and J. Ma, "Dendrimer-paclitaxel complexes for efficient treatment in ovarian cancer: study on OVCAR-3 and HEK293T cells," Acta Biochimica Polonica, vol. 65, no. 2, pp. 219-225, 2018.

[4] H. Ye, X. J. Liu, Y. Hui, Y. H. Liang, C. H. Li, and Q. Wan, "USF1 gene polymorphisms may associate with the efficacy and safety of chemotherapy based on paclitaxel and prognosis in the treatment of ovarian cancer," Neoplasma: Journal of Experimental and Clinical Oncology, vol. 65, no. 1, pp. 153160, 2018.

[5] L. Guo, S. Zhang, C. Chen, H. Zeng, F. Li, and Q. Xu, "Lentinan combined with cisplatin and paclitaxel in the treatment of patients with ovarian cancer with ascites," $E u$ ropean Journal of Gynaecological Oncology, vol. 39, no. 4, pp. 615-620, 2018.

[6] I. Alagkiozidis, C. Gorelick, T. Shah et al., "Synergy between paclitaxel and anti-cancer peptide PNC-27 in the treatment of ovarian cancer," Annals of Clinical and Laboratory Science: 
Official Journal of the Association of Clinical Scientists, vol. 47, no. 3, pp. 271-281, 2017.

[7] J. Zhai, R. B. Luwor, N. Ahmed et al., "Paclitaxel-loaded selfassembled lipid nanoparticles as targeted drug delivery systems for the treatment of aggressive ovarian cancer," ACS Applied Materials \& Interfaces, vol. 10, no. 30, pp. 2517425185, 2018.

[8] I. Vergote, G. Scambia, D. M. O'malley et al., “Trebananib or placebo plus carboplatin and paclitaxel as first-line treatment for advanced ovarian cancer (TRINOVA-3/ENGOT-ov2/ GOG-3001): a randomised, double-blind, phase 3 trial," The Lancet Oncology, vol. 20, no. 6, pp. 862-876, 2019.

[9] T. Luo, J. Sun, S. Zhu et al., "Ultrasound-mediated destruction of oxygen and paclitaxel loaded dual-targeting microbubbles for intraperitoneal treatment of ovarian cancer xenografts," Cancer Letters, vol. 391, pp. 1-11, 2017.

[10] X. Zhang, Y. Liu, Y. J. Kim, J. Mac, R. Zhuang, and P. Wang, "Co-delivery of carboplatin and paclitaxel via cross-linked multilamellar liposomes for ovarian cancer treatment," RSC Advances, vol. 7, no. 32, pp. 19685-19693, 2017.

[11] P. Tiet, J. Li, W. Abidi et al., "Silica coated paclitaxel nanocrystals enable neural stem cell loading for treatment of ovarian cancer," Bioconjugate Chemistry, vol. 30, no. 5, pp. 1415-1424, 2019.

[12] W. Shen, X. Chen, J. Luan, D. Wang, L. Yu, and J. Ding, "Sustained codelivery of cisplatin and paclitaxel via an injectable prodrug hydrogel for ovarian cancer treatment," ACS Applied Materials \& Interfaces, vol. 9, no. 46, pp. 40031-40046, 2017.

[13] M. Li, S. Zhang, Y. Ma, Y. Yang, and R. An, "Role of hsa-miR105 during the pathogenesis of paclitaxel resistance and its clinical implication in ovarian cancer," Oncology reports, vol. 45 , no. 5 , p. $84,2021$.

[14] E. Kadife, E. Chan, R. Luwor, G. Kannourakis, J. Findlay, and N. Ahmed, "Paclitaxel-induced src activation is inhibited by dasatinib treatment, independently of cancer stem cell properties, in a mouse model of ovarian cancer," Cancers, vol. 11, no. 2, p. 243, 2019.

[15] M. Świerczewska, A. Klejewski, M. Brązert et al., "New and old genes associated with primary and established responses to paclitaxel treatment in ovarian cancer cell lines," Molecules (Basel, Switzerland), vol. 23, no. 4, 2018.

[16] M. Yang, J. Zou, H. Zhu et al., "Paris saponin II inhibits human ovarian cancer cell-induced angiogenesis by modulating NF- $\kappa \mathrm{B}$ signaling," Oncology Reports, vol. 33, no. 5, pp. 2190-2198, 2015.

[17] I. Hussain, Waheed, Sana et al., "Scutellaria baicalensis targets the hypoxia-inducible factor- $1 \alpha$ and enhances cisplatin efficacy in ovarian cancer," Journal of Cellular Biochemistry, vol. 119, no. 9, pp. 7515-7524, 2018.

[18] L. Xu, L. Guo, H. Li, and X. Bian, "Hedyotis diffusa willd. extracts inhibit the proliferation and migration of ovarian cancer cells by modulating the AKT/ERK signaling pathway," Current Topics in Nutraceutical Research, vol. 17, no. 4, pp. 451-456, 2019.

[19] L. Zhao, Z. Liu, X. Deng et al., "Polyphyllin VII induces mitochondrial apoptosis by regulating the PP2A/AKT/DRP1 signaling axis in human ovarian cancer," Oncology Reports, vol. 45 , no. 2, pp. 513-522, 2021.

[20] W. Lin and Z. Li, "Blueberries inhibit cyclooxygenase-1 and cyclooxygenase-2 activity in human epithelial ovarian cancer," Oncology Letters, vol. 13, no. 6 Pt, pp. 4897-4904, 2017.

[21] G. Yin, D. Tang, J. Dai et al., "Combination efficacy of Astragalus membranaceus and Curcuma wenyujin at different stages of tumor progression in an imageable orthotopic nude mouse model of metastatic human ovarian cancer expressing red fluorescent protein," Anticancer Research: International Journal of Cancer Research and Treatment, vol. 35, no. 6, pp. 3193-3207, 2015.

[22] H. Yan, S. Xin, H. Wang, J. Ma, H. Zhang, and H. Wei, "Baicalein inhibits MMP-2 expression in human ovarian cancer cells by suppressing the p38 MAPK-dependent NF- $\kappa \mathrm{B}$ signaling pathway," Anti-Cancer Drugs, vol. 26, no. 6, pp. 649-656, 2015.

[23] J. Guoqiang, L. Jia, R. Baoyin et al., "Anti-tumor effects of osthole on ovarian cancer cells in vitro," Journal of Ethnopharmacology: An Interdisciplinary Journal Devoted to Bioscientific Research on Indigenous Drugs, vol. 193, pp. 368-376, 2016.

[24] B. Jiwon, L. Dahae, K. Tae et al., "O-( $\alpha$-l-rhamnopyranosyl) lyoniresinol from Lespedeza cuneata suppresses ovarian cancer cell proliferation through induction of apoptosis," Bioorganic and Medicinal Chemistry Letters, vol. 28, no. 2, pp. 122-128, 2018.

[25] T. Yiming and X. Huijun, "Effect of dioscorea pills on immunity of mice receiving chemotherapy for ovarian cancer," Open Access Library Journal, vol. 6, no. 2, pp. 1-10, 2019. 